Rev. Elév. Méd. vét. Pays trop. 1967, 20, 2 (329-342)

\title{
Comportement saisonnier du bétail Zébu en Adamaoua Camerounais I. Étude des femelles adultes: Comparaison de la race locale aux métis demi-sang Brahma
}

\author{
por P. LHOSTE
}

\begin{abstract}
RÉSUMÉ
L'auteur a étudié pendant 1 an l'interaction des facteurs écologiques et physiologiques sur le comportement des vaches de la station de Wakwa au Cameroun.

Deux populations généfiquement différentes ont été comparées : 110 vaches de race locale Foulbé et 134 vaches $1 / 2$ sang Brahma $\times$ Foulbé.

Des conclusions ont été dégagées :

10 La croissance pondérale de la vache continue jusqu'à 8-9 ans.

$2^{\circ}$ L'alternance saisonnière se traduit, en élevage extensif, par de grandes variations de poids de la vache; certans animoux subissent en période de disette une perte de $1 / 3$ de leur poids et sont très éprouvés.

30 L'époque de l'année à laquelle la vache met bas semble être un facteur déterminant de son comportement pondéral.

$40 \mathrm{Il}$ y a intérêt en pratique à regrouper les vêlages au début de la saison des pluies.
\end{abstract}

\section{I. - INTRODUCTION — GÉNÉRALITÉS}

Dans un contexte d'élevage extensif, l'observation des vaches reproductrices au cours d'une année complète en zone intertropicale nous montre que le comportement de ces animaux est très variable; certaines vaches passent à travers l'alternance saisonnière sans difficultés particulières, d'autres subissent en saison défavorable un amaigrissement alarmant qui peut même compromettre l'avenir de certains animaux.

Ces différences de comportement sont dues essentiellement à des combinaisons différentes des facteurs écologiques (saison, alimentation), avec les facteurs physiologiques propres à l'animal (gestation, mise bas, lactation).

Facteurs écologiques.

Nous rappelons que le milieu considéré se caractérise par alternance d'une saison des pluies et d'une saison sèche annuelles. Ce rythme détermine annuellement une période alimentaire pléthorique pour le bétall, suivie d'une période défavorable ; en Adamaoua, la période favorable se situe approximativement d'avril à octobre : la période défavorable d'octobre à mars, avec un maximum d'intensité en fin de saison sèche (février-mars).

Facteurs physiologiques propres à l'animal.

Nous rappelons que les besoins alimentaires de la vache sont rigoureusement fonction de son état physiologique. En plus de ses besoins normaux d'entretien et de croissance, la vache doit satisfaire à certaines époques des besoins supplémentaires de Gestation et de Lactation. Dans le contexte étudié ( Z Zébu d̀ viande » en Elevage extensif) (1) on peut admettre que les besoins maxima surviennent chez la vache au cours de la lactation: ces besoins de lactation peuvent alors représenter chez une bonne mère (4 à 51 de lait), l'équivalent des besoins d'entretıen. Ce qui revient à dıre qu'une vache «assez bonne laitière » en pleine lactation devra, pour maintenir son état, manger deux fois plus du 
même aliment qu'une vache de même poids tarie et non gestante.

Nous nous proposons d'étudier ci-après, les effets sur la vache de l'interaction des facteurs écologiques et des facteurs physiologiques. Cette étude porte sur les reproductrices de la Station de WAKWA où nous avons suivi mensuellement ou bı-mensuellement le poids de tous nos animaux, et cecl depuis plus d'un an. L'élevage considéré est de type extensif, les animaux étant entretenus sur le pâturage naturel sans complémentation alimentaire.

Notre étude portera d'autre part sur deux populations génétiquement différentes que nous comparerons. II s'dgit de :

- la Race locale Foulbé (ou «PeuleAdamaoud $\gg$;

- et de la Population Demi-Sang Brahma Issue du Métissage de première Génération entre la race locale et la race Brahma importée des Etats-Unis. Nos observations portent sur 134 Vaches Demi-Sang Brahma ef sur 110 Vaches de race locale.

\section{II. - POIDS ADULTE}

Pour chaque vache, nous avons d'abord établi un poids moyen annuel basé sur six pesées bimensuelles, régulièrement échelonnées dans l'année.

Les populations étudiées sont composées de vaches d'âge de 4 d̀ 12 ans, allant de la première à la huitième gestation. Ces deux groupes sont comparables par leur composition. Chaque groupe comprend trois troupeaux :

1 Troupeau de Primipares : Foulbé $=$ 36 Vaches. Demi-Sang $=46$ Vaches.

1 Troupeau de Vaches d'âge moyen : Foulbé $=$ 37 Vaches. Demi-Sang $=47$ Vaches $(2$ à 4 Vêlages).

1 Troupeau de Vaches âgées: Foulbé $=$ 37 Vaches. Demi-Sang $=41$ Vaches $($ de 4 à 8 Vêlages).

L'étude statistıque de la distribution des poids moyens annuels par vache dans ces deux populations donne les résultats suivants :

TABLEAU No I. - Distribution des poids mayens :

Vaches demi-sang brahma - vaches foulbé (classes de $10 \mathrm{~kg}$ )

\begin{tabular}{|c|c|c|c|c|}
\hline \multirow{2}{*}{$\begin{array}{l}\text { Milieu } \\
\text { des } \\
\text { classes kg }\end{array}$} & \multicolumn{2}{|c|}{ Vaches dem1-sang } & \multicolumn{2}{|c|}{ Vaches foulbé } \\
\hline & Effectifs & $\begin{array}{c}\text { Fréquences } \\
\text { E:p. } 100\end{array}$ & Effectifs & $\begin{array}{c}\text { Frëquences } \\
\mathrm{f}: \mathrm{p} \cdot 100\end{array}$ \\
\hline $\begin{array}{l}290 \\
300 \\
310 \\
320 \\
330 \\
340 \\
350 \\
360 \\
370 \\
380 \\
390 \\
400 \\
410 \\
420 \\
430 \\
440 \\
450 \\
460 \\
470 \\
480 \\
490\end{array}$ & $\begin{array}{r}2 \\
- \\
5 \\
7 \\
7 \\
9 \\
20 \\
20 \\
13 \\
10 \\
8 \\
10 \\
8 \\
6 \\
5 \\
4\end{array}$ & 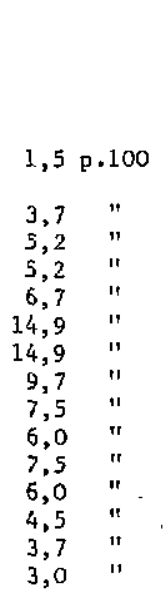 & $\begin{array}{r}1 \\
4 \\
5 \\
6 \\
21 \\
7 \\
15 \\
10 \\
11 \\
5 \\
5 \\
10 \\
2 \\
2 \\
1 \\
- \\
2 \\
- \\
- \\
2 \\
1\end{array}$ & 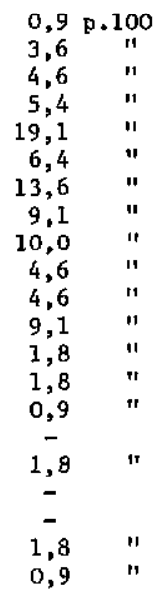 \\
\hline Effectif total & $n=134$ & $100 \mathrm{p} .100$ & $\mathrm{n}=110$ & $100 \mathrm{p} .100$ \\
\hline Moyenne $\bar{x}$ & $\bar{x}=41 B$ & & $\bar{x}=358$ & \\
\hline Erreur standard Sm & \pm 3 & & \pm 4 & \\
\hline Ecart-Type o & $\sigma=34$ & & $\sigma=39$ & \\
\hline Coefficient Variation & $v=8 p 100$ & & $\mathrm{~V}=11_{\mathrm{p}} 100$ & \\
\hline
\end{tabular}


La différence observée entre les moyennes de ces deux distributions est hautement significative (P. 0,01). Au graphique 1, nous donnons une représentation de ces distributions en présentanf les polygones des fréquences pour les deux populations.

Les fréquences ont été calculées en pourcentage pour permettre une comparaison plus facile entre les deux distributions.

Nous observons donc sur les moyennes générales, une différence de $60 \mathrm{~kg}$ en faveur de la vache Demı-Sang Brahma. Cette différence illustre l'amélioration très sensible de format et de poids apportée par la race Brahma. Cette supériorité du poids adultes'accompagne également d'un gain de précocité pour les animaux Métis.
Nous ajoutons que les vaches Foulbé de la Station constituent un échantillon très sensiblement supérieur à la moyenne régınale; ces animaux proviennent pour la plupart d'achats locaux, mais ils ont été l'objet d'une sélection assez sévère lors de mise à la reproduction.

Une analyse plus fine de l'évolution du poids adulte nous montre que la croissance pondérale de la vache se poursuit au-delà de l'âge de cınq ans. Nous produisons au Tableau 2, des chiffres moyens pour des classes d'âge successives. Les chiffres proposés pour les vaches Métis Brahma sont précis car il s'agit d'animaux nés sur la Station dont nous connaissons l'âge exact. Les chiffres proposés pour les vaches Foulbé sont indicatifs, car is résultent de l'estimation de l'âge d'anımaux d'achat.

TABLEAU $\mathrm{N}^{\circ} \mathrm{II}$

Poids moyen de la vache adulte (en fonction de l'âge)

\begin{tabular}{|l|c|c|c|c|c|r|r|r|r|}
\hline & Age & 4 ans & 5 ans & 6 ans & 7 ans & 8 ans & 9 ans & $10-12$ ans & Moyerne \\
\cline { 2 - 10 } $\begin{array}{l}\text { Vaches demf-sang } \\
\text { Brahma }\end{array}$ & Nombre & 7 & 21 & 16 & 19 & 25 & 40 & 6 & 134 \\
\hline Vaches foulbé & Polds & 372 & 400 & 405 & 423 & 413 & 436 & 435 & 418 \\
\hline
\end{tabular}

Malgré le caractère approximatif de la courbe des vaches Foulbé, nous constatons au Graphique 2 que les deux groupes d'animaux suivent une évolution parallèle. La différence de $60 \mathrm{~kg}$ en faveur des vaches Demi-Sang Brahma observée sur les moyennes générales semble se retrouver dans les différentes classes d'âge.

La conclusion qui découle de ce Graphıque est la suivante : chez la vache zébu, le poids maximum n'est pas atteint avant l'âge de 9 ans. La croissance pondérale est importante jusqu'à l'âge de 7 ans, pour devenır très lente audelà de 7 ans.

\section{III. - ÉVOLUTION PONDÉRALE SAISONNIÈRE}

Les conditions alimentaires dans lesquelles sont placés les animaux en élevage extensif sont très variables avec la saıson; les pluies déterminent la croissance du fourrage et sa qualité. L'alternance des saisons occasionne donc des variations de poids très importantes.
Au Tableau 3, nous présentons l'évolution pondérale saisonnière moyenne pour :

- Les Vaches Demi-Sang Brahma âgées (plus de 7 ans).

- Les Vaches Demi-Sang Brahma jeunes (moins de 7 ans).

- Toutes les Vaches Demi-Sang Brahma (Moyenne Générale).

- Les Vaches Foulbé âgées (plus de 7 ans).

- Les Vaches Foulbé jeunes (moins de 7 ans).

- Toutes les Vaches Foulbé (Moyenne Générale).

Nous remarquons en comparant l'évolution pondérale moyenne de chaque groupe (DemiSang Brahma-Foulbé) que la différence observée de $60 \mathrm{~kg}$ se retrouve pour chaque mois à $10 \mathrm{~kg}$ près.

Les deux groupes suivent donc une évolution très comparable au cours de l'année (Graphique $\left.n^{0} 3 \mathrm{~A}\right)$. 
DISTRIBUTIONS COMPAREES DES POIDS

Voches FOULBE Voches Demi-Song BRAHMA

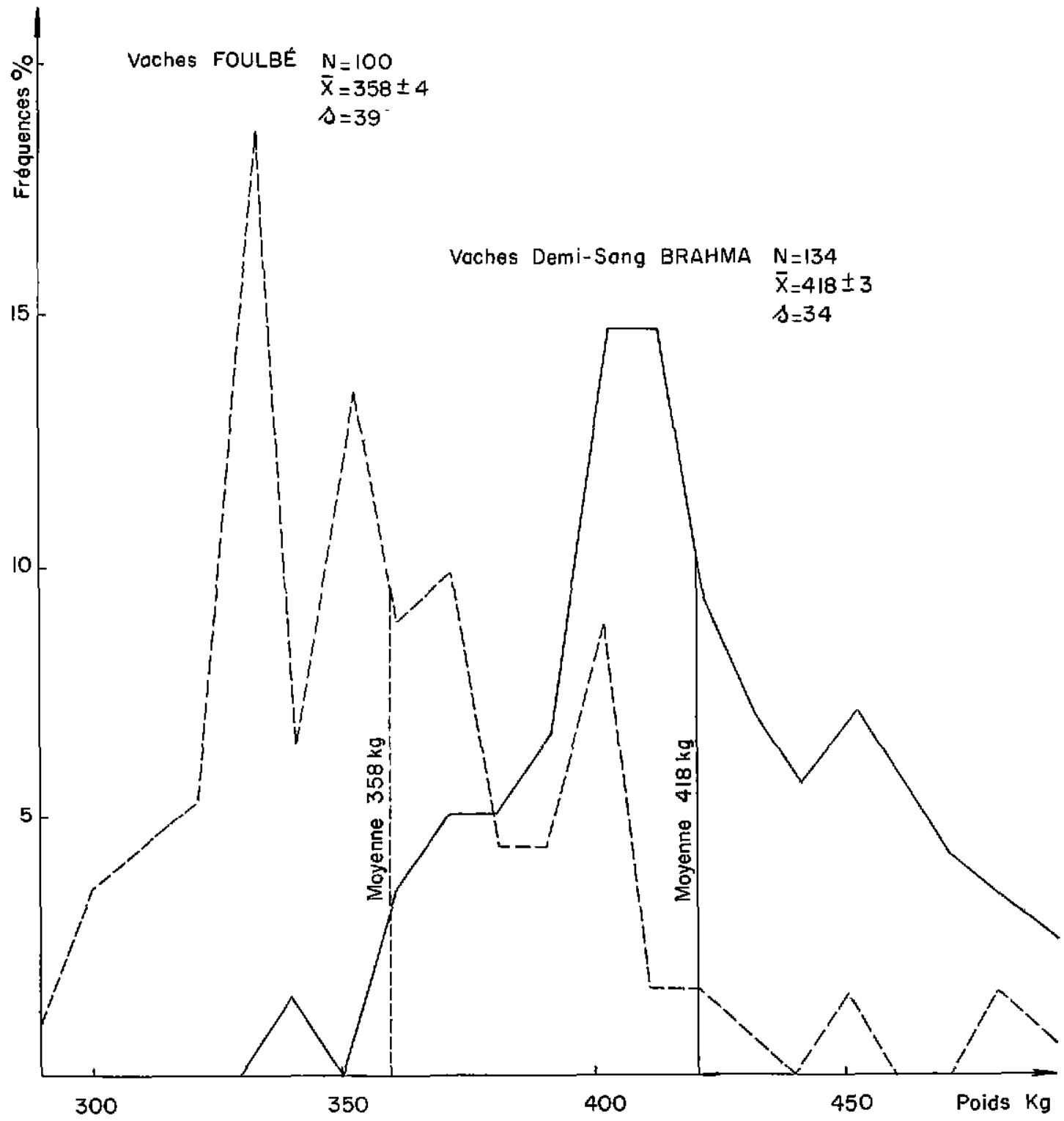


POIDS MOYEN VACHE ADULTE (en fonction de l'age)

Vaches FOULBÉ Vaches Demi-Sang BRAHMA

Poids kg

\section{LÉGENDE}

Demi-Sang BRAHMA

FOULBE

343 Poids Moyen

73 Nb d'animoux

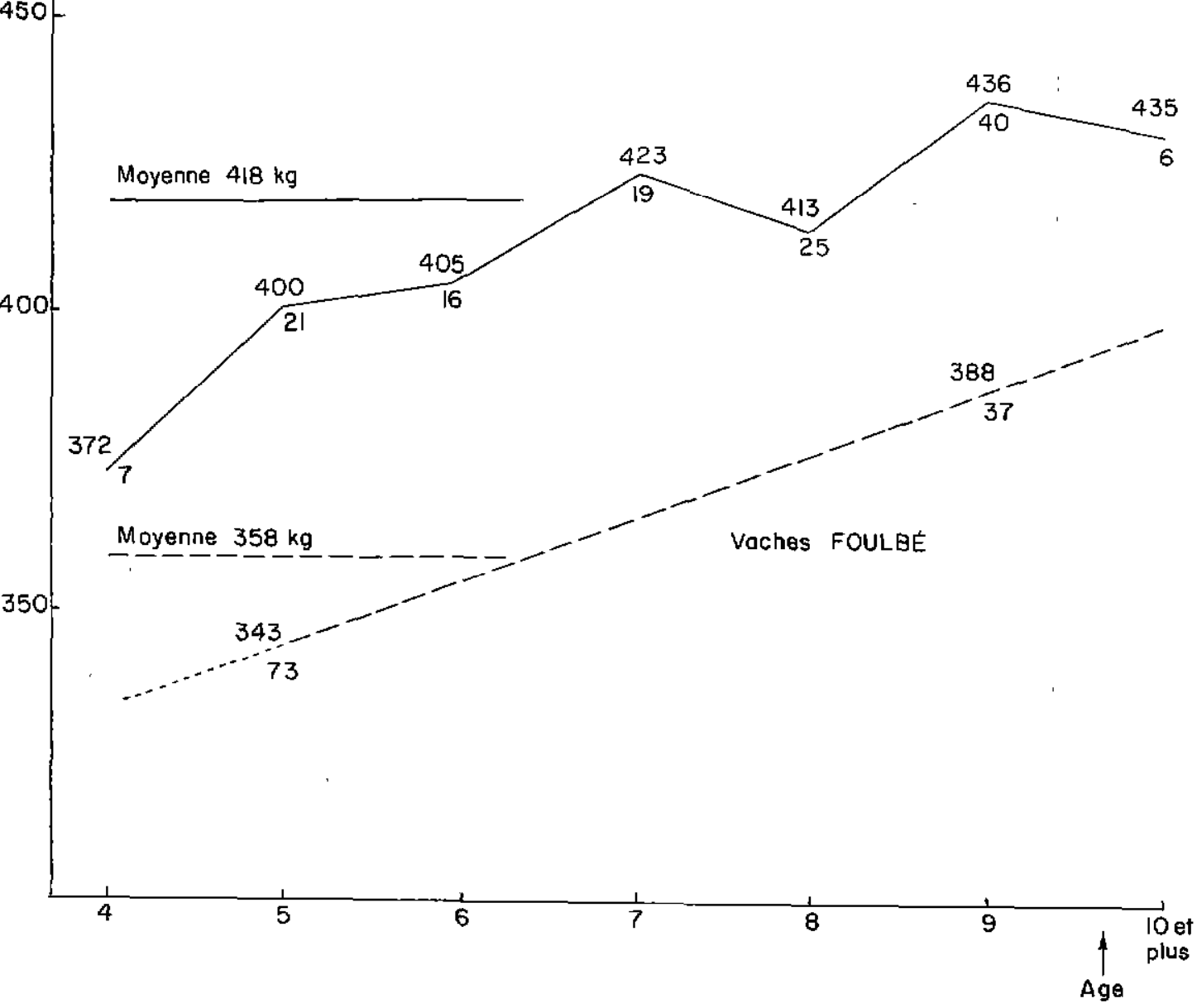


TABLEAU No III. - Évolution pondérale saisonnière

\begin{tabular}{|c|c|c|c|c|c|c|c|c|c|c|c|c|c|}
\hline & \multirow{2}{*}{$\begin{array}{c}\text { Nombre } \\
\text { de } \\
\text { vaches }\end{array}$} & \multicolumn{11}{|c|}{ Dates des pesé es } & \multirow[b]{2}{*}{ Moyenne } \\
\hline & & $\begin{array}{r}10 / \\
64\end{array}$ & $\begin{array}{r}12 / \\
64\end{array}$ & $\begin{array}{l}21 \\
65\end{array}$ & $\begin{array}{l}4 / \\
65\end{array}$ & $\begin{array}{l}61 \\
65\end{array}$ & $\begin{array}{l}7 / \\
65\end{array}$ & $\begin{array}{l}8 / \\
65\end{array}$ & $\begin{array}{l}9 / \\
65\end{array}$ & $\begin{array}{r}10 / \\
65\end{array}$ & $\begin{array}{r}11 / \\
65\end{array}$ & $\begin{array}{r}12 / \\
65\end{array}$ & \\
\hline \multicolumn{14}{|l|}{ Vaches demi-sang brahma } \\
\hline Agées & 90 & 455 & 437 & 398 & 381 & 438 & 452 & 461 & 479 & 476 & 462 & 439 & $42 B$ \\
\hline Jeunes & 44 & 409 & 389 & 361 & 361 & 417 & 425 & 448 & 466 & 466 & 446 & 430 & 397 \\
\hline Tota1 & 134 & 439 & 421 & 386 & 375 & 432 & 444 & 456 & 475 & 473 & 457 & 436 & 418 \\
\hline \multicolumn{14}{|l|}{ Vaches foulbẽ } \\
\hline Agées & 37 & 421 & 389 & 355 & 343 & 404 & 415 & 417 & 433 & 436 & $40 B$ & 387 & 388 \\
\hline Jeunes & 73 & 346 & 349 & 328 & 313 & 353 & 366 & 374 & 388 & 392 & 330 & 352 & 344 \\
\hline Total & 110 & 371 & 362 & 337 & 323 & 369 & 383 & 388 & 404 & 407 & 382 & 364 & 358 \\
\hline
\end{tabular}

Les courbes produites sont approximativement parallèles et présentent une allure sinusoidale de période 1 an, et d'amplitude $100 \mathrm{~kg}$ environ.

Le poids minimum est observé à la pesée d'avril, mais se situe en falt en mars (d'après d'cutres études) ; le poids maximum est observé en septembre ou octobre.

Au Tableau 3, nous avons produit également l'évolution comparée des jeunes vaches ef des vaches âgées pour chaque groupe (DemiSang Brahma-Foulbé). Graphıque n० 3 B). Nous constatons que les jeunes anımaux semblent mieux résister aux conditions défavorables, et de plus mieux récupérer lorsque les conditions alimentaires s'améliorent.

Ceci s'explique par le fait que les vaches de moins de sept ans ont encore une croissance pondérale appréciable qui atténue les effets néfastes de la saison défavorable, mais qui accentue la récupération pondérale en pérıde d'alimentation abondante.

Pendant la période de récupération maximum (avril d̀ juin), certains animaux prennent plus d'un kg par jour, soit un taux de l'ordre de $3 \mathrm{~g} / j o u r / \mathrm{kg}$ poids vif. Les moyennes s'établıssent ainsi :

\begin{tabular}{|c|c|c|c|c|}
\hline Période avrit-juin & $\begin{array}{c}\text { Croit } \\
\text { brut } \\
\mathrm{kg}\end{array}$ & $\begin{array}{c}\text { Croît } \\
\text { journal } \\
\mathrm{kg} / \mathrm{j}\end{array}$ & $\begin{array}{c}\text { Crôt relatif } \\
\text { g////kg } \\
\text { Poids vif }\end{array}$ \\
\hline $\begin{array}{c}\text { Moyennes } \\
\text { demi-sang }\end{array}$ & 134 vaches & 57 & 0,93 & 2,5 \\
$\begin{array}{c}\text { Moyennes } \\
\text { faulbe..... }\end{array}$ & 110 vaches & 46 & 0,76 & 2,4 \\
\hline
\end{tabular}

Le poids de la vache est donc soumis à d'importantes variations saisonnières. L'état physiologique de l'animal au cours de ces saisons constıtue le deuxième facteur important du comportement de la vache, et c'est l'objet de l'étude du chapitre suivant.

\section{IV. - INTERACTION DE L'ÉPOQUE DU VÊLAGE ET DE L'ÉVOLUTION PONDÉRALE DES VACHES}

Nous avons observé ci-dessus, l'amplitude moyenne des variations pondérales annuelles de la vache zébu. L'amplitude de cette variation est de l'ordre de $100 \mathrm{~kg}$, ce qui est déjà considérable pour des animaux de $400 \mathrm{~kg}$ en moyenne. $\mathrm{Ce}$ chiffre n'est qu'un résultat moyen. Nous remarquons en effet que le comportement des vaches est variable d'un animal à l'autre, en raison des différentes époques de vêlage dans l'année. Le poids et l'étał de l'animal résultent en effet de la satısfaction de ses besoins alimentaires (variables avec l'état physiologique : croissance, gestation, lactation...), compte tenu des disponibilités alımentares (variables avec la saison).

La situation dans l'année de la période de vêlage par rapport aux saisons apparaît donc comme un facteur primordial dont nous analysons ci-dessous les répercussions.

Nous étudierons ce facteur en regroupant les vaches observées en quatre groupes en fonction de l'époque de vêlage :

1) Vêlage de début de saison sèche (15 octobre-15 février). 
Retour au menu

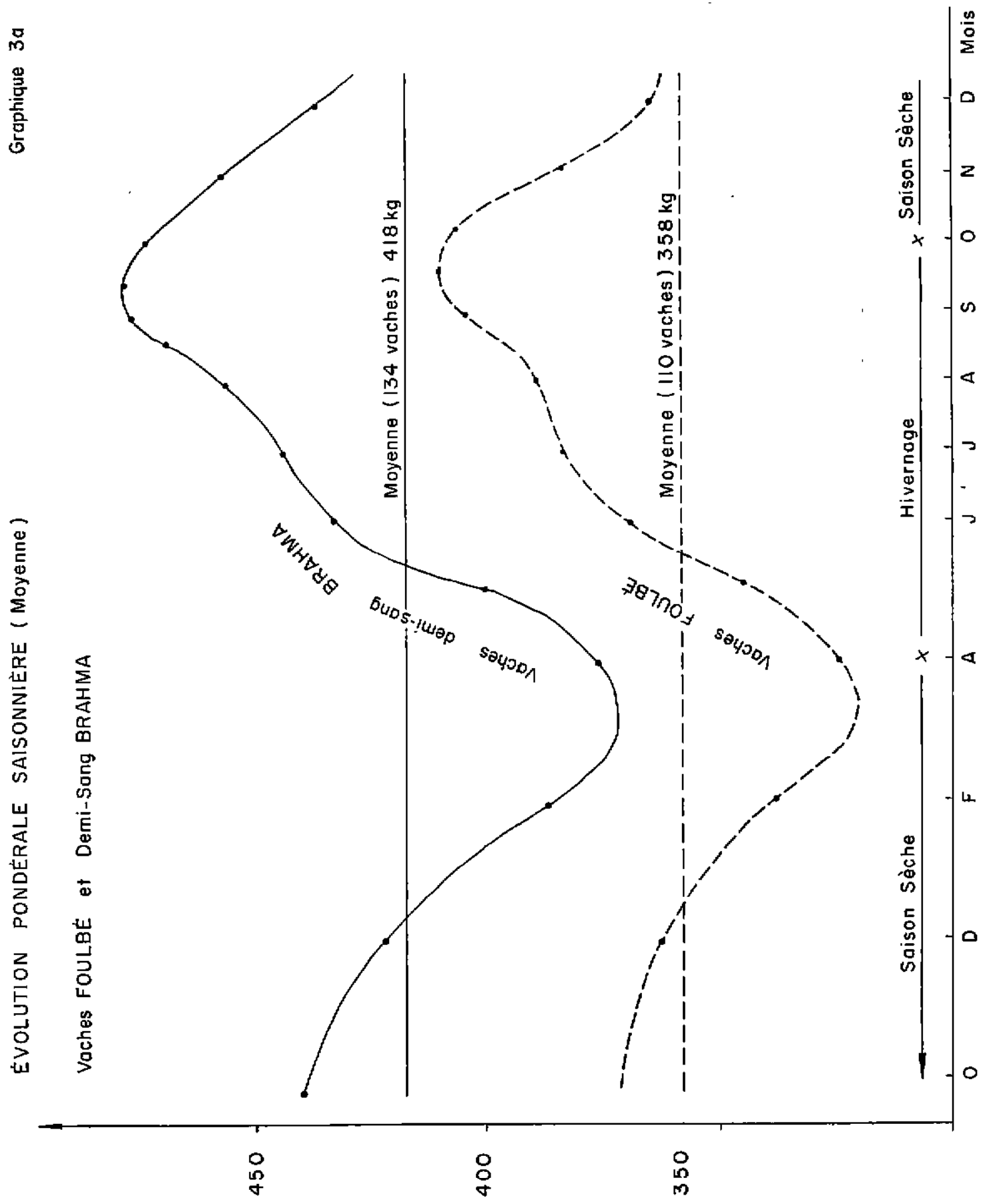

335 
EVOLUTION PONDERALE COMPAREE

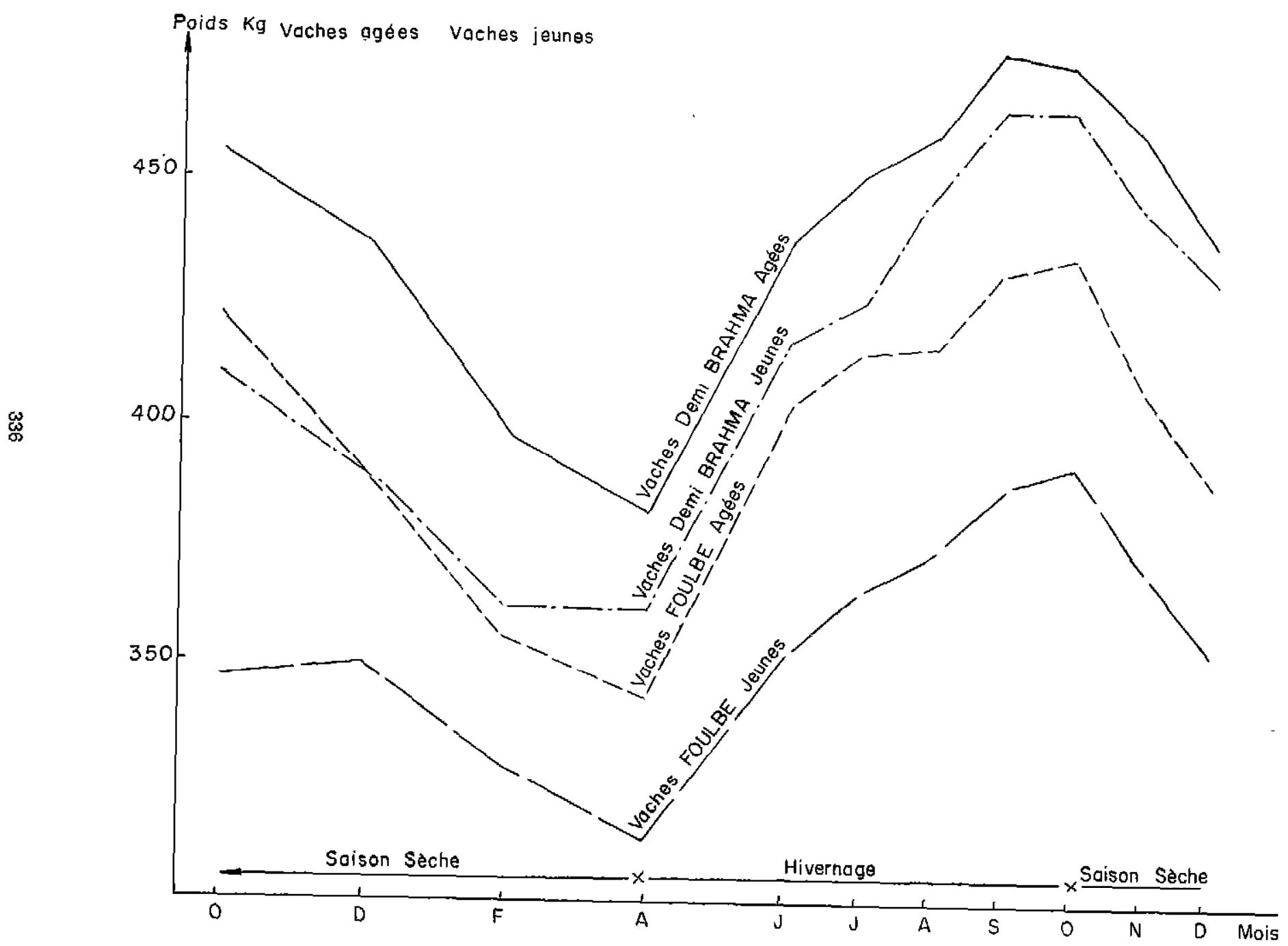


TABLEAU $N^{\circ} I V$

Evolution pondérale des vaches foulbé et demi-sang brahma

(groupées en 4 périodes de vêlage)

\begin{tabular}{|c|c|c|c|c|c|c|c|c|c|c|c|c|c|}
\hline \multirow{2}{*}{\multicolumn{2}{|c|}{ Pérlade de vêlage }} & \multirow{3}{*}{$\begin{array}{c}\begin{array}{c}\text { Nombre } \\
\text { de } \\
\text { vaches }\end{array} \\
23\end{array}$} & \multicolumn{11}{|c|}{ Poids moyen (kg.) et indice de pourcentage aux dates } \\
\hline & & & \multirow{2}{*}{$\begin{array}{c}10 / 64 \\
468 \\
100 \mathrm{p} 100\end{array}$} & \multirow{2}{*}{$\begin{array}{c}12 / 64 \\
436 \\
93 \mathrm{p} 100\end{array}$} & \multirow{2}{*}{$\begin{array}{c}2 / 65 \\
371 \\
79 \mathrm{p} 100\end{array}$} & \multirow{2}{*}{$\begin{array}{c}4 / 65 \\
358 \\
76 \mathrm{p} 100\end{array}$} & \multirow{2}{*}{ 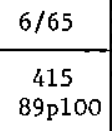 } & \multirow{2}{*}{ 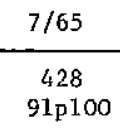 } & \multirow{2}{*}{ 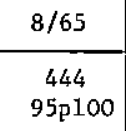 } & \multirow{2}{*}{ 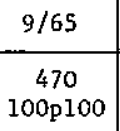 } & \multirow{2}{*}{$\begin{array}{c}10 / 65 \\
471 \\
101 p 100\end{array}$} & \multirow{2}{*}{ 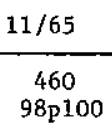 } & \multirow{2}{*}{$\begin{array}{c}12 / 65 \\
450 \\
96 \mathrm{p} 100\end{array}$} \\
\hline $\begin{array}{l}\text { Début } \\
\text { salson sếche }\end{array}$ & Demi-sang & & & & & & & & & & & & \\
\hline$(15-10$ a $15-2)$ & Foulbé & 14 & $100^{432}$ & $\begin{array}{l}386 \\
89\end{array}$ & $76^{328} "$ & $72^{313}$ & ${ }^{381}$ & $91^{395} "$ & $93^{402}$ & $9^{424}$ & $\begin{array}{l}429 \\
99^{\prime \prime}\end{array}$ & $93^{409} "$ & $90^{387} "$ \\
\hline $\begin{array}{l}\text { Fin } \\
\text { saitaon sèche }\end{array}$ & & 23 & $\begin{array}{r}448 \\
100^{\prime \prime}\end{array}$ & 944 & $94^{420}$ & $81^{361}$ & $9_{93}^{418} "$ & $96^{431}$ & $\begin{array}{l}440 \\
98^{\prime \prime}\end{array}$ & ${ }_{103}^{461} "$ & ${ }_{103}^{460} "$ & $9^{443} "$ & ${ }_{95}^{426} "$ \\
\hline$(15-2$ a $15-4)$ & Foulbé & 6 & $\begin{array}{r}420 \\
100 " 1\end{array}$ & $9^{416} "$ & $\begin{array}{c}387 \\
92^{\prime \prime}\end{array}$ & $75^{317}$ & $92^{387} "$ & $95^{398} "$ & 908 & $101^{426} "$ & $104^{435}$ " & $98^{412} "$ & $95^{399} 1$ \\
\hline $\begin{array}{l}\text { Début } \\
\text { satson des plutes }\end{array}$ & & 35 & $\begin{array}{r}432 \\
100 "\end{array}$ & $9^{418} "$ & $92^{397}$ & $\begin{array}{l}402 \\
93^{\prime \prime}\end{array}$ & 101 & $101^{435} 1$ & $104^{449} "$ & $108^{467}$ & ${ }^{468}$ & $103^{447} "$ & $\begin{array}{l}425 \\
98^{\prime \prime}\end{array}$ \\
\hline$(15-4$ a $15-7)$ & Foulbẽ & 51 & $100^{356}$ & $101^{360} "$ & $97^{345} "$ & $94^{334} "$ & $101^{358} "$ & $103^{366} "$ & $105^{373} "$ & $108^{385} "$ & $108^{385} "$ & $101^{360}$ & $96^{340} "$ \\
\hline $\begin{array}{l}\text { Fin } \\
\text { saison des pluies }\end{array}$ & & 30 & $\begin{array}{r}438 \\
100^{\prime \prime}\end{array}$ & $910 "$ & $85^{373} "$ & $86^{375}$ & $103^{451 "}$ & $108^{472 "}$ & $108^{475} "$ & $111^{484} "$ & $107^{468} "$ & $102^{447}$ & 920 \\
\hline$(15-7$ à $15-10)$ & Foulbē & 20 & $\begin{array}{r}388 \\
100 " 1\end{array}$ & $96^{371 "}$ & $89^{345} "$ & ${ }^{338} 7^{\prime \prime}$ & 104 & ${ }^{423}$ " & $106^{410}$ & $107^{416}$ & ${ }^{408}{ }^{\prime \prime}$ & $99^{383} "$ & $92^{357}$ " \\
\hline $\begin{array}{l}\text { Totaux } \\
\text { moyenne générale }\end{array}$ & Demi-sang & 111 & $\begin{array}{r}444 \\
100 " 1\end{array}$ & $96^{425} "$ & $88^{390} "$ & $8^{377}$ & $97^{432} "$ & $100^{443} "$ & ${ }^{453} "$ & $106^{471}$ & $105^{467}$ & $101^{449} "$ & 929 \\
\hline & Foulbë & 91 & $\begin{array}{r}379 \\
100^{\prime \prime}\end{array}$ & $\begin{array}{l}370 \\
98\end{array}$ & $91^{345} 1$ & $\begin{array}{c}331^{\prime \prime} \\
87^{\prime \prime}\end{array}$ & $9^{373} "$ & $102^{385} "$ & $102^{388} "$ & ${ }_{106}^{401 "}$ & $106^{401 "}$ & $100^{378}$ & $94^{355} "$ \\
\hline
\end{tabular}




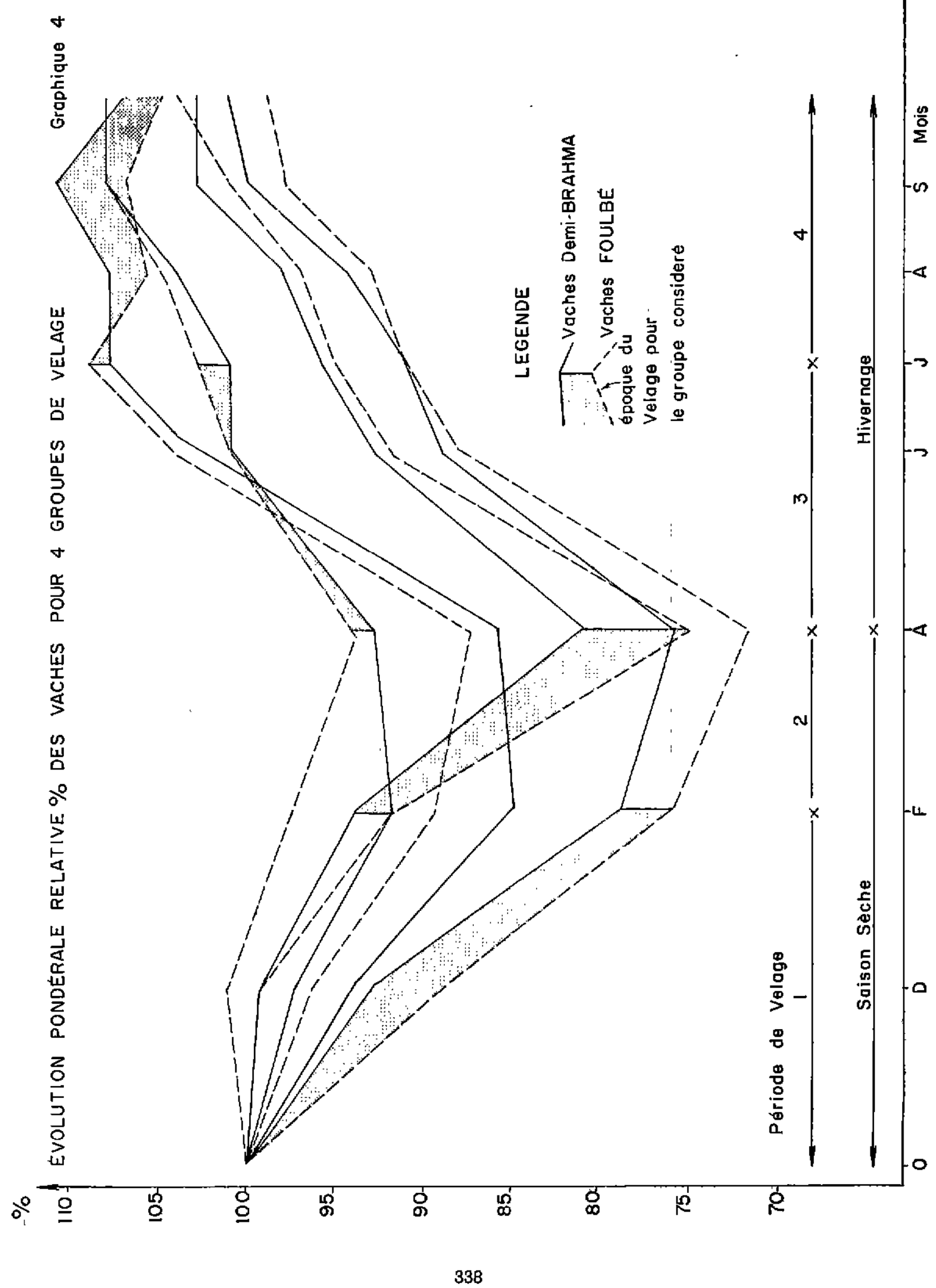


2) Vêlage de fin de saison sèche (15 février15 avril).

3) Vêlage de début de saıson des pluies (15 avril-15 juin).

4) Vêlage de fin de saison des pluies (15 juillet-15 octobre).

Nous présentons au Tableau 4, l'évolution pondérale annuelle des vaches Foulbé et des vaches Demi-Sang Brahma pour chaque groupe de vêlage.

Nous avons porté d'une part les poids moyens établis d'après l'ensemble de nos observations, et'd'autre part pour chaque mois le poids relatif calculé en pourcentage du polds de départ (octobre 1964). Cet indice (p. 100) nous permet de comparer le comportement des deux groupes (Foulbé-Demi-Sang Brahma), en annulant les différences de format (et de poids) entre les animaux.

Un premier examen du Tableau 4 nous montre qu'il existe une grande différence de comportement entre les vaches des différent́s groupes de vêlage. Il existe en revanche une grande similitude d'évolution pondérale relative entre les vaches Demi-Sang Brahma et les vaches Foulbé d'un même groupe de vêlage. Ceci est mis en évidence au Graphique $n^{0} 4$ dans lequel nous avons produit les courbes d'évolution pondérale relative (p.100) pour les deux groupes génétiques de vaches, et pour les 4 périodes de vêlage. Nous y remarquons immédiatement un appariement très net des courbes (Foulbé et Demi-Sang) deux à deux, pour chaque période de vêlage.

$\mathrm{Si}$ nous reprenons successivement chaque période de vêlage, nous pouvons faıre les remarques suivantes :

fer Groupe : Vêlages de début de saison sèche (octobre à février). Les vêlages à cette époque de l'année sont les plus éprouvants pour les vaches; la mère se trouve en effet en pleine lactation (besoins alımentaires maximum) au cours de la saison la plus défavorable (fin de scison sèche).

Dans ce groupe, on observe des pertes de poids de 24 p. 100 pour les vaches Demi-Sang. et de 28 p. 100 pour les Foulbé ; ces chiffres moyens nous donnent une idée de ce que représente l'amaigrissement des animaux les moins favorisés, qui peut atteindre pour certains individus le tiers du poids de base.

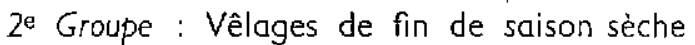
(février à avril). Les vêlages à cette époque sont encore éprouvants pour la mère qui, doit assurer le début de sa lactation en saison défavarable ; mais l'amaigrıssement est moıns grave que pour le groupe précédent et la récupération de la vache est satisfaisante car la pointe, de lactation se produit alors lorsque les conditions alimentaires se sont améliorées.

On observe dans ce groupe des pertes de poids de 19 p. 100 pour les Demi-Sang Brahma et de 25 p. 100 pour les vaches Foulbé.

3e Groupe : Vêlages de début de saison des pluies (mai à juillet). Les vaches qui mettent bas au début de l'hivernage sont de toute évidence les moins éprouvées. Certes, la fin de la gestation en saison sèche est quelque peu épulsante, mais ce qui est essentiel, la lactation a lieu lorsque le fourrage est abondant et de bonne qualité.

Nous observons pour ces vaches (Cf. Graphique $n^{\circ} 4$ ), une courbe d'évolution pondérale très peu anguleuse en comparaison des autres groupes. Les pertes de poids en salson sèche sont minimes (7 p. 100 et 6 p. 100), et la récupération est très satisfaısante.

4e Groupe : Vêlages de fin de saison des pluies (août à octobre). Les vêlages de fin de saison des pluies déterminent un comportement intermédiaire pour ce groupe de vaches. Les mères vêlent à l'époque la plus favorable et sont donc peu traumatisées par la mıse bas, mais elles doivent assurer la fin de leur lactation lorsque les conditions alimentarres se sont déjà détériorées.

En conclusion : Cette étude nous a permis de mettre en évidence l'effet de l'époque du vêlage sur le comportement de la vache-mère. Nous pouvons ainsi dégager dans l'année deux grandes périodes:

- ¿'ovril à octobre : Période conseillée pour les mises bas (avec optimum en avril-mai);

- de novembre à mars : Période déconseillée pour les mises bas (et plus spécialement janvierfévrier). 


\section{V. - COMPARAISON DES APTITUDES DES VACHES FOULBE ET DEMI-SANG BRAHMA}

Après avoir dégagé les grandes lignes du comportement de la vache-mère, il est intéressant de se pencher sur les différences qui peuvent exister entre les deux groupes génétiques observés : Demı-Sang Brahma-Foulbé.

Nous avons étudié au Chapitre II ci-dessus, la différence globale de format entre les deux groupes, qui s'exprime approxımativement par une supériorité de poids de $60 \mathrm{~kg}$ pour la vache Demı-Sang Brahma.

Pour étudier les différences de comportement de ces deux groupes, nous pouvons nous reporter aux indices relatıfs (p. 100) du Tableau 4; nous élımınons ainsi l'ınfluence de la différence de format. Au Graphique 4, nous pouvons aussi constater visuellement les différences de compor- tement des vaches pour une même période de vêlage.

Pour préciser ces différences, nous calculons :

- Le coefficient moyen d'amaigrissement (période d'octobre à avril), en g/jour $/ \mathrm{kg}$. Polds Vif.

- Le coefficient moyen de récupération (période d'avril à octobre) en $\mathrm{g} / \mathrm{jour} / \mathrm{kg}$. Poids Vif.

- L'amplitude annuelle de variation de poids en p. $100 \mathrm{du}$ poids de base (octobre).

Ces données sont calculées pour chaque groupe de vêlage, puisque nous avons vu au chapitre précédent que l'époque du vêlage constıtue le facteur déterminant du comportement de la vache.

Nous présentons au Tableau 5 ci-dessous, les différentes données pour les deux groupes génétıques étudiés, ef pour les 4 époques de vêlage.

TABLEAU $\mathbb{N}^{\circ} \mathrm{V}$

Variations de poids des vaches foulbé et demi-sang brahma

(en g/jour $/ \mathrm{kg}$. Poids vif et en pourcentage du poids base)

\begin{tabular}{|c|c|c|c|c|}
\hline \multicolumn{2}{|c|}{ Epoque de vêlage } & $\begin{array}{c}\text { Coefficient } \\
\text { d'anaigrissement } \\
\text { (octobre à avrli) }\end{array}$ & $\begin{array}{l}\text { Coefficient } \\
\text { de rêcupération } \\
\text { (avrî à octobre) }\end{array}$ & $\begin{array}{c}\text { Amplitude } \\
\text { annuelle de } \\
\text { variation de poids }\end{array}$ \\
\hline $\begin{array}{l}\text { Début } \\
\text { saison sèche } \\
\text { octobre-février }\end{array}$ & $\begin{array}{l}23 \text { demi-sang } \\
14 \text { foulbe }\end{array}$ & $\begin{array}{l}1,3 \mathrm{~g} / \mathrm{jour} / \mathrm{kg} \\
1,5 \mathrm{~g} / \mathrm{jour} / \mathrm{kg}\end{array}$ & $\begin{array}{l}1,8 \mathrm{~g} / \mathrm{j} \text { our } / \mathrm{kg} \\
2,1 \mathrm{~g} / \mathrm{jour} / \mathrm{kg}\end{array}$ & $\begin{array}{l}25 p \cdot 100 \\
28 " ~\end{array}$ \\
\hline $\begin{array}{l}\text { Fin } \\
\text { saison sèche } \\
\text { février-avri1 }\end{array}$ & $\begin{array}{l}23 \text { demi-sang } \\
6 \text { foulbé }\end{array}$ & $\begin{array}{l}1,1 \mathrm{~g} / \mathrm{J} \text { our } / \mathrm{kg} \\
1,4 \mathrm{~g} / \mathrm{j} \text { our } / \mathrm{kg}\end{array}$ & $\begin{array}{l}1,5 \mathrm{~g} / \mathrm{jour} / \mathrm{kg} \\
2,1 \mathrm{~g} / \mathrm{jour} / \mathrm{kg}\end{array}$ & $\begin{array}{ll}22 & " 1 \\
29 & \end{array}$ \\
\hline $\begin{array}{l}\text { Début } \\
\text { hivernage } \\
\text { mai-juillet }\end{array}$ & $\begin{array}{l}35 \text { demi-sang } \\
51 \text { foulbé }\end{array}$ & $\begin{array}{l}0,4 \mathrm{~g} / \mathrm{J} \text { our } / \mathrm{kg} \\
0,3 \mathrm{~g} / \mathrm{jour} / \mathrm{kg}\end{array}$ & $\begin{array}{l}0,9 \mathrm{~g} / \mathrm{jour} / \mathrm{kg} \\
0,8 \mathrm{~g} / \mathrm{jour} / \mathrm{kg}\end{array}$ & $\begin{array}{l}16 " ~ \\
14 " ~\end{array}$ \\
\hline $\begin{array}{l}\text { Fin } \\
\text { hivernage } \\
\text { août-octobre }\end{array}$ & $\begin{array}{l}30 \text { demi-sang } \\
20 \text { foulbe }\end{array}$ & $\begin{array}{l}0,8 \mathrm{~g} / \mathrm{jour} / \mathrm{kg} \\
0,7 \mathrm{~g} / \mathrm{jour} / \mathrm{kg}\end{array}$ & $\begin{array}{l}1,4 \mathrm{~g} / \text { jour } / \mathrm{kg} \\
1,1 \mathrm{~g} / \text { jour } / \mathrm{kg}\end{array}$ & $\begin{array}{ll}25 & " \\
20 & "\end{array}$ \\
\hline
\end{tabular}

L'examen des Tableaux 4 ef 5 nous amène à formuler les remarques suivantes :

10 En période de vêlage défavorable (saison sèche) : Nous observons un comportement plus satisfaisant pour les vaches Demi-Sang que pour les vaches Foulbé. L'amaigrissement relatif est en effet significativement plus faible pour les Demi-Sang. Les deux groupes de vêlage en saison défavorable (début ef fin de saison sèche) confirment ces résultats. Cette supériorité des Demi-Sang se retrouve nettement sur les courbes du Graphique no 4.
Nous en concluons que les produits Métis Brahma résistent mieux aux conditions alimentaires défavorables que les animaux de race locale. C'est un argument important en faveur de cette race, car il est rare d'observer chez une race importée, une adaptation aux conditions du milıeu supérieure à celle de la race locale.

Notons en revanche que sur le plan pathologique, l'adaptation de la race Brahma n'est pas satisfassante : comme nous l'avons montré dans une étude partıculıère, le métis Brahma se révèle notamment très sensible d̀ la Streptothricose (2). 
$2^{\circ}$ En période de vêlage favorable (Hıvernage): Nous observons pour ces groupes, une supériorité apparente de la race locale pour laquelle l'amaigrissement serait légèrement inférieur à celui des Demi-Sang Brahma. Mais un examen plus approfondi des échantillons comparés nous montre que le groupe des vaches Foulbé (51 têtes) comprenait 28 vaches au premier vêlage, contre seulement 2 Primıpares chez les vaches DemiSang (35 têtes au total). Cette grande proportion de jeunes vaches chez les Foulbé confère à ce groupe un comportement partıculier, car la croissance des animaux jeunes tend à atténuer partiellement les effets défavorables de la saison.

Nous pouvons donc conclure pour cette période de vêlage favorable à un comportement sensiblement identique entre les deux groupes comparés.

$3^{\circ}$ L'amplitude annuelle de variation de poids nous donne une idée des difficultés que rencontre l'animal au cours de l'année. On constate en effet après un an d'expérience (en octobre 1965), que le poids moyen des animaux esł passé à 105 p. 100 de ce qu'il était au début du contrôle (en octobre 1964); cette augmentation moyenne de 5 p. 100 est répartie sur l'ensemble des observations, mais elle est due surtout aux plus jeunes vaches en crolssance.

Or, au cours de cette année de contrôle, certaıns anımaux ont subi des variations de poids qui représentent plus du tiers de leur poids initial. En examinant les moyennes d'amplitude annuelle de variation de poids, nous remarquons que les vaches les moins éprouvées sont celles dont le vêlage a lieu au début de l'hivernage : ceci confirme les résultats du chapitre précédent.

Pour le groupe des anımaux vêlant en saıson défavorable, on constate chez les vaches Foulbé, des amplitudes moyennes de variation pondérale de 28 et 29 p. 100 contre 25 et 22 p. 100 pour les Demi-Sang. Les Métis Brahma apparalssent donc mains sensibles aux effets défavorables de la saison, ils supportent mieux la saison sèche que les animaux de race locale.

\section{VI. - CONCLUSIONS}

Cette étude de la vache reproductrice zébu nous permet de dégager quelques conclusions :

- La croissance pondérale de la vache se poursuit jusqu'à l'âge de $8-9$ ans; jusqu'à 7 ans, cette croissance demeure assez importante ; audelà de 7 ans, elle est lente.

- En zone intertropicale, les vaches conduites en Elevage extensif sont soumises au cours de l'année d̀ des conditions alimentaires très variables; cette alternance sassonnière d'une période d'alımentation satisfaisante (hıvernage) avec une période déficitaire (saison sèche) se traduit par de grandes variations du poids des vaches. Certains anımaux peuvent perdre au cours de la saison défavorable le tiers de leur poids maximum. Cet amaıgrissement est très éprouvant pour l'animal.

- L'époque de l'année à laquelle la vache met bas nous appraraît être le facteur déterminant du comportement pondéral de la vache. Il est important pour maintenir la vache en état correct de farre coïncider sa lactation (besoins maxımum) avec la période favorable d'alimentation.

Cette étude nous permet de formuler une règle pratıque concernant le calendrier de conduite du troupeau :

\section{Règle pratique :}

Dans la mesure du possible, Il y a intérêt à regrouper les vêlages au début de la saison des pluies. Il est important d'éviter les mises bas de début de saison sèche qui sont les plus éprouvantes pour les reproductrices.

Pratıquement, il y a un intérêł zotechnique certain à instıtuer quand cela est possible, une «saison de monte » qui aura pour effet de déterminer une «saison de naissance» au moment favorable. La durée de cette saison de monte peut être de 6 ou 8 mois; en Adamaoua, elle commencerait en juin pour se termıner en décembre ou février. On évite ainsi les vêlages les plus défavorables, et on provoque un maxımum des naissances en début d'hıvernage.

Cette étude nous a permis également de comparer deux populations génétiquement différentes : la race locale « Foulbé » (ou Peule-Adamaoua »), et la population Métis Demi-Sang Brahma.

Au point de vue format, l'introduction du sang Brahma se traduit par une supériorité pondérale de l'ordre de $60 \mathrm{~kg}$ chez les vaches Demi-Sang, les poids moyens étant de $358 \mathrm{~kg}$ pour les vaches Foulbé, et de $418 \mathrm{~kg}$ pour les vaches DemiSang Brahma. 
Sur le plan de l'évolution pondérale, la résistance ef l'adapatation aux conditions de milieu se révêlent aussi supérieures pour les Métis Brahme. En période défavorable, tout particulièrement, les vaches Demi-Sang Brahma maigrissent moins que les vaches de race locale.

La race Brahma a donc été amélioratrice pour le format ef pour l'adaptation aux conditions alimentares du milieu étudié.

\section{SUMMARY}

Response of zebu in Adamawa (Cameroon) to the seasonal climatic variations.

I. Study of the adult females : Comparison between the local breed and the metis $1 / 2$ breed Brahma

The influence of some ecological and physiological factors on the weight of the cows of Wakwa's station in Cameroon has been studied during a year.

Two populations genetically different have been compared :

110 cows of the local Foulani breed and 134 cows $1 / 2$ breed Brahma $\times$ Foulani. Following conclusions have been recorded :

10 The ponderal growth of the cow occured up to $8-9$ years.

$2^{\circ}$ In extensive rearing the alternations of the seasons resulted in great weight variations of the cow ;

Some animals showed, in undernutrition period, a loss of $1 / 3$ of their weight and had severely suffered.

$3^{0}$ The season of calving seems to be an influent factor of the weight variation.

40 Actually, it is interesting that calving occurs early in the rainy season.

\section{RESUMEN}

Comportamiento del ganado cebú según la estación en Adamaua (Camerún).

I. Estudio de las hembras adultas :

Comparación de la raza local con los mestizos $1 / 2$ sangre Brahma

Durante un año, el autor estudió la interacción de los factores ecologicos y fisiológicos en el comportamiento de la vacas de la estación de Wakwa en el Camerún. Se compararon dos populaciones diferentes desde el punto de vista de la genética : 110 vacas de raza local Fulbé y 134 vacas $1 / 2$ sangre Brahma $\times$ Fulbé. Se ha concluido lo sıguiente:

10 El peso de la vaca aumentaba hasta 8-9 años.

$2^{\circ}$ Las importantes variaciones del peso de las vacas en ganaderia extensiva resultan de la alternación ajustada a estación ; durante el periodo de escasez, algunos animales pierden $1 / 3$ de su peso y su salud está muy mala.

$3^{\circ}$ La época de la parición parece ser un factor determinante de las madificaciones de peso de las vacas.

40 Prácticamente se necesita grupar los partos al principio de la eslación de las lluvias.

\section{BIBLIOGRAPHIE}

1. DUMAS (R.) et LHOSTE (P.). - Voriations du poids vif ef du rendement en viande de bceufs zébus de l'Adamaoua au cours de la saison sèche. Rev. Elev. Méd. Vét. Pays trop., $1966,19,4: 573-79$.
2. Rapport annuel 1965, Centre de Recherches Zootechniques et station fourragère de Wakwa. I. E. M. V. T. 